\title{
Robotic mitral repair for Barlow's disease with bileaflet prolapse and annular calcification using pericardial patch technique
}

\author{
Didier F. Loulmet ${ }^{1}$, Michael S. Koeckert ${ }^{1}$, Peter J. Neuburger ${ }^{2}$, Robert Nampiaparampil ${ }^{2}$, Eugene A. Grossi ${ }^{1}$ \\ ${ }^{1}$ Departments of Cardiothoracic Surgery, ${ }^{2}$ Departments of Anesthesiology, NYU Langone Medical Center, New York, NY, USA \\ Correspondence to: Eugene A. Grossi, MD. 530 First Avenue, Suite 9V, New York, NY 10016, USA. Email: eugene.grossi@nyumc.org.
}

Submitted Dec 20, 2016. Accepted for publication Jan 10, 2017.

doi: 10.21037/acs.2017.01.09

View this article at: http://dx.doi.org/10.21037/acs.2017.01.09

\section{Clinical vignette}

A 58-year-old asymptomatic and otherwise healthy woman presented for surgical evaluation after echocardiography revealed severe mitral valve regurgitation (MR). She had a family history of MR and was being followed by her primary care doctor for a murmur. Transthoracic echocardiography (TTE) demonstrated severe MR due to bileaflet prolapse, Barlow's disease, a left atrial dimension of $44 \mathrm{~mm}$, a left ventricular end systolic dimension of $36 \mathrm{~mm}$, and a preserved ejection fraction. Mitral annular calcification (MAC) was also identified. After careful evaluation we believed that the extent of the MAC in this patient could be handed with the robotic technique as we have done before. There was no other significant valvular disease and the patient did not have coronary disease or arrhythmias.

\section{Surgical techniques}

\section{Preparation}

Preoperative evaluation included computed tomography angiography of the chest, abdomen and pelvis, along with coronary angiography. The patient was placed supine on the operative table with the right hemi-thorax elevated 30 degrees; the right arm was supported below the level of the chest. The left femoral vessels were exposed through a $3-\mathrm{cm}$ incision and cannulae were placed using Seldinger technique and echocardiographic guidance. Venous drainage was obtained with a 25 -French long cannula and the femoral artery was cannulated with a 21 -French EndoReturn arterial cannula through which an EndoClamp (Edwards Lifesciences, Irvine, California, USA) was inserted. The EndoClamp is our preference as it is an elegant and simple solution for this totally endoscopic approach; it avoids both making another chest wall incision and the necessity of placing a needle in the ascending aorta for cardioplegia and venting.

\section{Exposure}

Three $8-\mathrm{mm}$ instrument ports and one $12-\mathrm{mm}$ camera port were placed in the right thorax and a 2 -cm work port (fourth interspace) was created without rib spreading using a soft tissue retractor. The breast was pushed medially to avoid any trocar placement through breast tissue (Figure 1). The da Vinci Xi system (Intuitive Surgical; Sunnyvale, CA, USA) was then docked, cardiopulmonary bypass (CPB) initiated, and the right lung collapsed. The pericardium was opened longitudinally, anterior to the phrenic nerve, and stay sutures were placed to improve exposure. The Endoballon provided aortic clamping and was used to deliver antegrade cardioplegia. The left atrium was then entered through Sondergaard's groove, the mitral valve was exposed with a robotic septal retractor, and a vent was placed in the left inferior pulmonary vein.

\section{Operation}

While completing cardioplegia delivery, a patent foramen ovale was closed and the left atrial appendage was oversewn (Video 1). Analysis of the intra-operative transesophageal echocardiography (TEE) demonstrated prolapse of P2 due to elongated and ruptured chordae as well as prolapse of $\mathrm{P} 3$. There was a large area of MAC at the level of the hinge of P2 and P3. The base of these leaflets were detached from their annular hinge and the annular calcification was excised. The resultant defect between the left ventricle free wall and the left atrium was reconstructed with a bovine pericardial patch. 


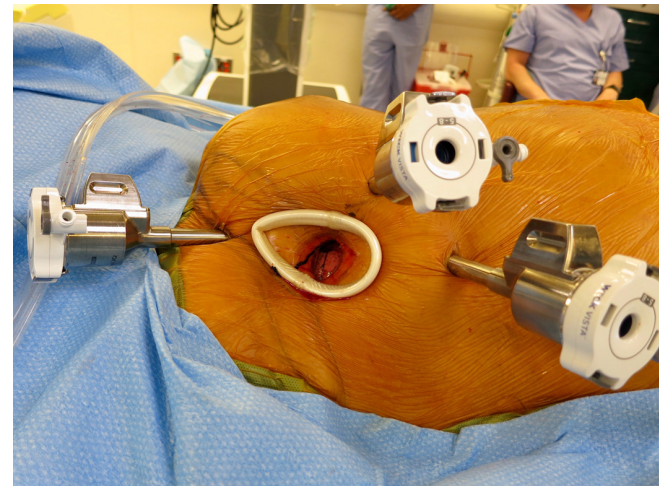

Figure 1 Port placement and medial displacement of breast mound.

Excessive leaflet from the base of $\mathrm{P} 2$ and $\mathrm{P} 3$ was excised; leaflet mobilization enhanced by dividing secondary chordae. The bases of $\mathrm{P} 2$ and $\mathrm{P} 3$ where re-suspended with running 4-0 polytetrafluoroethylene (PTFE) suture to the neo-annulus. The neo-annulus was therefore comprised of atrial edge, bovine pericardial patch, and leaflet base. The remaining cleft between of $\mathrm{P} 2$ and $\mathrm{P} 3$ was closed with a running 5-0 PTFE suture. The posterior commissure was also reconstructed with a running PTFE suture. The mitral valve repair was completed with an annuloplasty using a Medtronic CG Future band size $34-\mathrm{mm}$. The band was sutured to the posterior aspect of the mitral orifice using a series of 2-0 polyester horizontal mattress sutures. The knots were tied with a Ti-Knots (LSI Solutions). The valve was tested by injecting saline into the left ventricle; the line of closure was favorable and there was no residual regurgitation.

\section{Completion}

The EndoClamp was deflated with suction on the aortic root and the pulmonary vein vent was repositioned passed the mitral valve in the ventricle. While warming was completed, the left atrium was closed with a running 2-0 Gore-Tex suture. When the absence of air in the left ventricular cavity was demonstrated on TEE, the left ventricular vent was removed and the heart was allowed to eject into the aorta. We placed a pacing wire on the right ventricle. The patient was weaned off $\mathrm{CPB}$ with ventricularpacing and some inotropic support. CPB time was 204 minutes with the EndoClamp applied for 164 minutes.

After removing the cannulae, heparin was reversed with protamine. The port sites were closed after placing a 28 -French chest tube into the right pleural cavity. The left groin incision was closed after closing the left femoral artery with a 6-0 polypropylene suture.

\section{Comments \\ Clinical results}

Immediate postoperative TEE demonstrated a successful repair. There was no residual regurgitation, systolic anterior motion or transvalvular gradient. The left ventricular function was comparable to the preoperative status. There were no intraoperative complications. The patient was discharged on post-operative day 6. TTE prior to discharge showed no residual MR.

\section{Advantages}

Totally endoscopic robotic techniques provide advantages for both surgeon and patient. The da Vinci system is both ergonomic and dexterous while providing excellent visualization of the mitral valve and the subvalvular region while avoiding sternotomy. Smaller incisions reduce operative stress, postoperative pain and hospital length-ofstay, while improving cosmesis and recovery time $(1,2)$.

\section{Caveats}

Appropriate patient selection and preoperative work-up are crucial for a successful robotic mitral valve repair. Suitability depends on chest anatomy and status of the vasculature. Preoperative computed tomography angiography can identify calcific and non-calcific atheroma, tortuosity, localized dissections and aberrant or small vessels. This enables the allowing selection of an appropriate cannulation strategy and helps avoid the potential complications of retrograde arterial perfusion $(3,4)$.

\section{Acknowledgements}

None.

\section{Footnote}

Conflicts of Interest: The authors have no conflicts of interest to declare.

\section{References}

1. Mihaljevic T, Jarrett CM, Gillinov AM, et al. Robotic repair 
of posterior mitral valve prolapse versus conventional approaches: potential realized. J Thorac Cardiovasc Surg 2011;141:72-80.e1-4.

2. Woo YJ, Nacke EA. Robotic minimally invasive mitral valve reconstruction yields less blood product transfusion and shorter length of stay. Surgery 2006;140:263-7.

3. Ailawadi G, Agnihotri AK, Mehall JR, et al. Minimally

Cite this article as: Loulmet DF, Koeckert MS, Neuburger PJ, Nampiaparampil R, Grossi EA. Robotic mitral repair for Barlow's disease with bileaflet prolapse and annular calcification using pericardial patch technique. Ann Cardiothorac Surg 2017;6(1):67-69. doi: 10.21037/acs.2017.01.09
Invasive Mitral Valve Surgery I: Patient Selection, Evaluation, and Planning. Innovations (Phila) 2016;11:243-50.

4. Ward AF, Loulmet DF, Neuburger PJ, et al. Outcomes of peripheral perfusion with balloon aortic clamping for totally endoscopic robotic mitral valve repair. J Thorac Cardiovasc Surg 2014;148:2769-72. 